\title{
Velocity measurements in the liquid metal flow driven by a two-phase inductor
}

\author{
A. Pedcenko - A. Bojarevičs . \\ J. Priede - G. Gerbeth - R. Hermann
}

the date of receipt and acceptance should be inserted later

\begin{abstract}
We present the results of velocity measurements obtained by ultrasonic Doppler velocimetry and local potential probes in the flow of GaInSn eutectic melt driven by a two-phase inductor in a cylindrical container. This type of flow is expected in a recent modification to the floating zone technique for the growth of small-diameter single intermetallic compound crystals. We show that the flow structure can be changed from the typical two toroidal vortices to a single vortex by increasing the phase shift between the currents in the two coils from $0^{\circ}$ to $90^{\circ}$ degrees. The latter configuration is thought to be favourable for the growth of single crystals. The flow is also computed numerically, and a reasonable agreement with the experimental results is found. The obtained results may be useful for the design of combined two-phase electromagnetic stirrers and induction heaters for metal or semiconductor melts.
\end{abstract}

\section{Introduction}

The present work is concerned with a physical modelling of the melt flow in a recent modification to the floating zone technique for the growth of smalldiameter single intermetallic compound crystals [10]. The quality of crystals grown by this method depends on the growth conditions, particularly on the

Coventry University, Applied Mathematics Research Centre, Priory Street, Coventry, CV1 5FB, UK . Institute of Physics, University of Latvia, Salaspils, LV-2169, Latvia . Coventry University, Applied Mathematics Research Centre, Priory Street, Coventry, CV1 5FB, UK · Helmholtz-Zentrum Dresden-Rossendorf, P.O. Box 510119, D-01314 Dresden, Germany - Leibniz Institute for Solid State and Materials Research (IFW) Dresden, Germany shape of the solidification front. The growth of single crystals usually requires a convex solidification front [13]. The shape of this front can strongly be affected by the convective heat transport in the melt. A standard single-phase induction heater usually drives a radially inward jet of hot melt at the middle of the floating zone [6]. At the centre of the floating zone, the radial jet splits into two nearly symmetric axial jets, which further stream towards both solid-liquid interfaces. The hot axial jet impinging at the centre of the growth interface renders it concave so promoting a polycrystalline growth. To overcome this adverse effect, we recently proposed a two-phase inductor, which is able to reverse the direction of the electromagnetically driven melt flow so that the solidification front becomes mostly convex [7].

The inductor consists of two coils that are fed by alternating currents of the same amplitude but a 90 degrees phase shift between them. In the original design for radio-frequency induction heating, the phase lag is achieved by short circuiting the secondary coil through a variable capacitor that is tuned so that the resonance frequency of the secondary circuit coincides with the frequency of the power supply [9]. In this way, a component of the magnetic field is created that travels towards the coil with the phase lag so dragging the liquid metal along it. As a result, the outer layers of the hot melt are driven towards the periphery of the solidification front while the cooled down melt returns in the centre [12]. A similar multiphase stirrer for low conductivity melts has been developed by Ernst et al. [3], who also used it for refining the grain size in gold alloys [4].

Until now, there have been no direct experimental observations of the effect of the phase shift in a two-coil inductor on the structure of ensuing fluid 
flow. In this paper, we present comprehensive results of velocity measurements in a model experiment using GaInSn eutectic alloy driven by a simple two-phase stirrer in a cylindrical container. The evolution of the flow pattern has been determined depending on the phase shift between the two coils which were connected to separate power supplies with externally controllable phase shifts. The experiments were also modelled numerically and numerical results compared with the measurements.

The paper is organized as follows. In the following two sections, experimental setup and measurement techniques are described. Experimental results are presented in Sec. 4 and compared with numerical results in Sec. 5. The paper is concluded by a summary in Sec. 6.

\section{Experimental setup}

The main element of the experimental setup shown in Fig. 11is an open top cylindrical container of $50 \mathrm{~mm}$ in both the diameter and height (unity aspect ratio) made of a $2 \mathrm{~mm}$-thick Polycarbonate. The container was filled with GaInSn alloy at eutectic composition with $67 \% \mathrm{Ga}, 20.5 \%$ In and $12.5 \% \mathrm{Sn}$, and kept in a water jacket at $15^{\circ} \mathrm{C}$ temperature. The relevant physical properties of GaInSn are listed in Table 1 . The top surface of the liquid metal was kept open for measurements. The Joule heat generated in the liquid metal was efficiently removed by passing the cooling water through a labyrinth of thin partitioning walls, which were uniformly distributed along the side wall of the container. The container was placed inside an inductor consisting of two circular copper coils of diameter $D_{\text {ind }}=88 \mathrm{~mm}$, axial separation $d=\frac{1}{2} D_{\text {ind }}$ and a rectangular $8 \times 9 \mathrm{~mm}^{2}$ crosssection with an inner cooling channel. To minimize the perturbation of the magnetic field by the coil terminals, the latter were made of a 2 mm-thick copper strip welded into each copper winding with a $1 \mathrm{~mm}$ gap, which was filled with Teflon. Thus the current in each coil was interrupted only over this small gap which constituted less than $0.4 \%$ of the total coil circumference. To minimize the superposition of perturbations, the terminals of each coil were placed across the diameter of the container as shown in Fig. 1b.

The coils were powered as follows. First, two source signals with preset amplitudes, frequencies and relative phases were generated digitally by a computer program and then converted into analogue form by a D/A converter. Second, the analogue signals were amplified by a two-channel power amplifier. Two toroidawidely employed technique for measuring velocities

\begin{tabular}{|c|c|}
\hline density & $\rho=6360 \mathrm{~kg} / \mathrm{m}^{3}$ \\
\hline electrical conductivity & $\sigma=3.2 \times 10^{6} \mathrm{~S} / \mathrm{m}$ \\
\hline kinematic viscosity & $\nu=3.4 \times 10^{-7} \mathrm{~m}^{2} / \mathrm{s}$ \\
\hline container diameter $=$ height & $H=D=0.05 \mathrm{~m}$ \\
\hline diameter of coils & $D_{\text {ind }}=0.088 \mathrm{~m}$ \\
\hline distance between coils & $d=0.044 \mathrm{~m}$ \\
\hline current amplitude & $I_{0}=100 \cdots 600 \mathrm{~A}$ \\
\hline AC frequency & $f=400 \mathrm{~Hz}$ \\
\hline
\end{tabular}

Table 1 Physical properties of the GaInSn alloy and experimental parameters.

transformers were used to match the output impedance of the amplifier with that of the inductor. This allowed us to reach the RMS current up to $600 \mathrm{~A}$ in each coil, which produced magnetic field with the amplitude of vertical component $B_{0}=17 \mathrm{mT}$ in the centre of inductor. As the phase shift between the currents in both coils was increased from $\Delta \phi=0^{\circ}$ to $90^{\circ}, 120^{\circ}, 150^{\circ}$ the amplitude decreased respectively to $B_{0} \approx 12,8,4.5 \mathrm{mT}$. At $\Delta \phi=180^{\circ}$ the vertical components of the magnetic field produced by the opposite currents in both coils nearly compensated each other in the mid-plane of the inductor. The magnetic field in the liquid metal was further reduced by the electromagnetic skin effect with the characteristic thickness $\delta=1 / \sqrt{\mu_{0} \sigma \omega / 2}$, where $\mu_{0}=$ $4 \pi \times 10^{-7} \mathrm{H} / \mathrm{m}$ is the vacuum permeability and $\omega=$ $2 \pi f$ is the circular AC frequency. For the AC frequency $f=400 \mathrm{~Hz}$ used in this experiment we had $\delta \approx 15 \mathrm{~mm}$. This value is close to the optimal one which produces maximal flow velocity at fixed current amplitude in this setup [8].

Typical melt velocity of $V=50 \mathrm{~mm} / \mathrm{s}$ corresponds to the Reynolds number $R e=D V / \nu \approx 7400$, which implies turbulent flow regime. Magnitude of the electromagnetic force is characterized by the dimensionless interaction parameter $N=\left(D I_{0} \mu_{0} / \nu\right)^{2} \omega \sigma / \rho(\approx$ $3.9 \times 10^{9}$ for $I_{0}=300 \mathrm{~A}$ ).

\section{Measurement techniques}

The velocity distribution in the liquid metal was measured by two methods: pulsed ultrasound Doppler velocimetry (UDV) and potential probe with local magnetic field, which both are briefly described below.

\subsection{Ultrasound Doppler velocimetry}

Ultrasound Doppler pulsed velocimetry (UDV) is a 


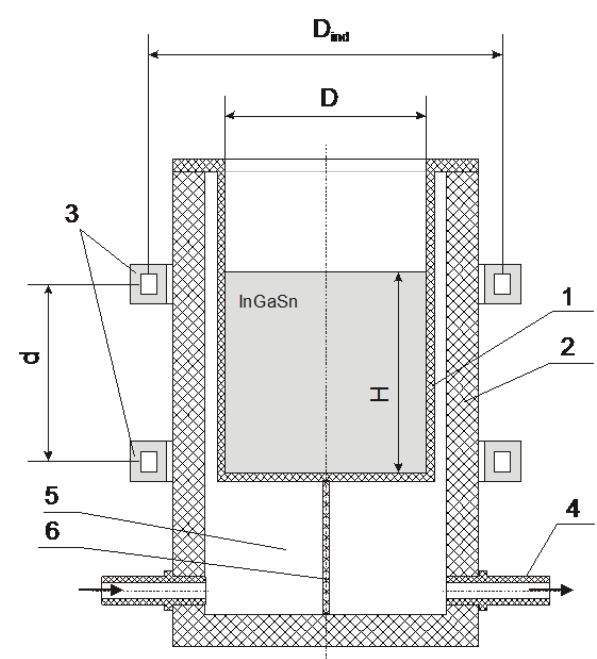

(a)

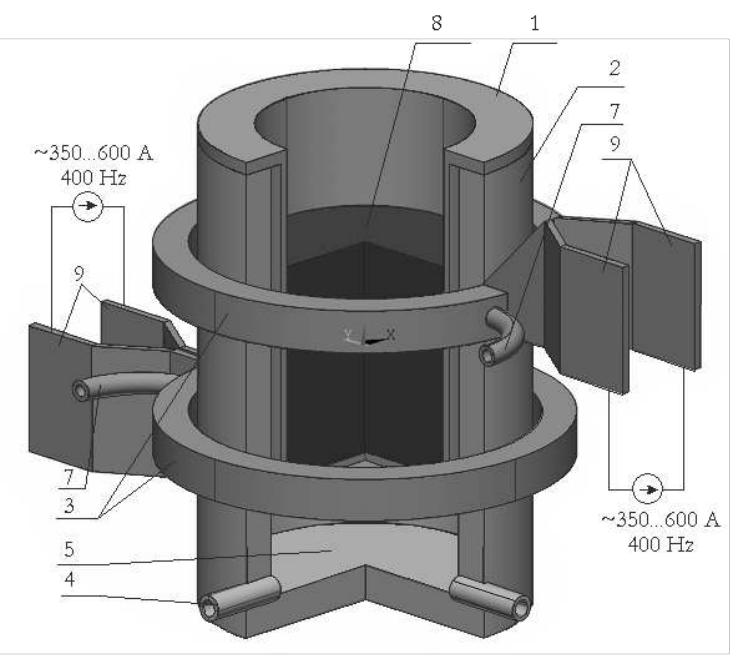

(b)

Fig. 1 Experimental setup in cross-section (a) and 3D (b) views showing the inner container of diameter $D=50 \mathrm{~mm}$ with liquid metal (1), the outer container with cooling water (2), two single-winding coils of diameter $D_{\text {ind }}=88 \mathrm{~mm}$ and vertical separation $d=44 \mathrm{~mm}$ (3), one of the six cooling water intakes and outlets distributed uniformly along the circumference (4), water jacket (5), cooling water partitioning walls (6), water intakes and outlets for coil cooling (7), GaInSn alloy (8), coil terminals (9).

in liquid metal flows [17,18,1]. The method uses the phase shift of the ultrasonic wave scattered by microscopic particles in the liquid to determine the distance to the particles. By analysing the time correlation of the signals acquired at different instants of time the device reconstructs the velocity of particles along the ultrasonic beam. In this study we employed DOP2000 Velocimeter model 2125 ('Signal Processing SA', Switzerland) to measure the axial velocity profiles along the height of the container in the flow of GaInSn driven by a two-phase AC magnetic field. Measuring velocities of a few centimetres per second over a relatively small height of the container required the highest available ultrasound frequency of $10 \mathrm{MHz}$. For the pulse repetition frequency of $1428 \mathrm{~Hz}$ used in the experiment and the speed of sound in the liquid metal of $2740 \mathrm{~m} / \mathrm{s}$, the velocity was measured with the absolute error not exceeding $1 \mathrm{~mm} / \mathrm{s}$.

The ultrasound wave was introduced into the liquid metal directly through its free surface. A special care was taken to ensure a good wetting of the UDV transducer by the liquid metal, which was essential for the acoustic coupling. The vertical velocity profiles were measured at the following 9 radial positions: $r=0, \pm 5, \pm 10, \pm 15, \pm 21 \mathrm{~mm}$. The radial size of the UDV probe precluded measurements at larger $r$, i.e., closer to the side wall. At each radial position, 300 samples of axial velocity profiles were recorded during a $35 \mathrm{~s}$ interval with $\approx 8.5 \mathrm{~Hz}$ sampling rate and a $0.3 \mathrm{~mm}$ spatial resolution in the axial direction. These data were used to find both the time av-

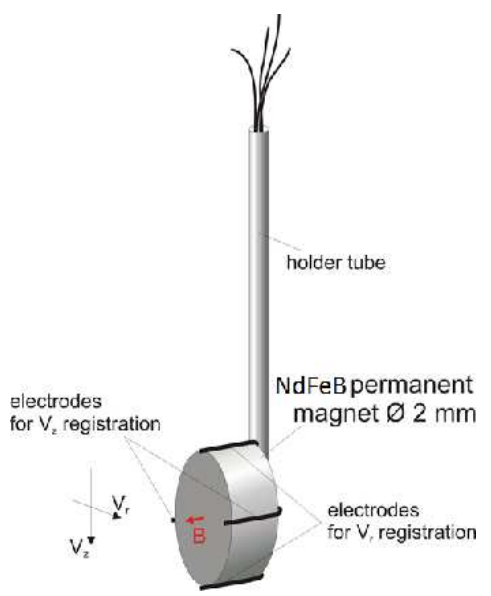

Fig. 2 Potential probe consisting of a small permanent magnet with two pairs of electrodes. The horizontal and vertical pairs of electrodes measure the opposite, that is the vertical and horizontal, respectively, velocity components.

erage of the velocity and its standard deviation, i.e. the intensity of velocity pulsations, at each of $9 \times 170$ spatial measurement points over the vertical crosssection of the container. Since the measurements at different $r$ were taken at different times, it was not possible to follow the temporal evolution of the flow over the whole cross-section.

\subsection{Potential probe}

Potential probe, also known as the Vives probe [11, [2], is another commonly used technique for local velocity measurements in low-melting-point liquid met- 
als. The probe shown in Fig. 2 consists of a small permanent magnet with two pairs of small electrodes. The liquid metal flow passing between the electrode pairs in the magnetic field of the probe induces a difference of the electric potential between the electrodes proportional to the velocity in the respective direction. Besides the low cost, the main advantage of the potential probe over a single-channel UDV is the possibility to measure simultaneously two components of local velocity with a high temporal sampling rate. However, there are also several disadvantages. First, very low voltages, typically about $0.1 \ldots 0.2 \mu \mathrm{V} / \mathrm{mm} / \mathrm{s}$, need to be measured. Second, quite long measurement times are required even at moderate spatial resolutions to acquire the velocity distribution over the whole cross-section of the container. Third, the temperature of the liquid metal has to be kept sufficiently uniform to avoid thermo-electrically induced potential perturbations. In the present experiment, the potential probe was used only for a few measurements to check the UDV results.

The probe used in this experiment to measure the axial and radial velocity components was made of a cylindrical $\mathrm{NdFeB}$ permanent magnet of $2 \mathrm{~mm}$ in both diameter and height, which was hold by a stainless steel tube of $1 \mathrm{~mm}$ in diameter. The probe was calibrated using an annular channel filled with GaInSn, which was rotated about its centre with a well-defined angular velocity. Since the sensitivity of the probe is nonlinear at low velocities (see Fig. 3), calibration was carried out for the whole range of the expected velocities. The calibration data were fitted with two mathematical expressions: a 'sigmoid law' for low velocities and a linear one for higher velocities. These expressions were further used in the acquisition software to convert the voltage measurements into velocities.

Measurements were taken at 121 locations in the vertical cross-section of the container. At each locationm the axial and radial velocity components were recorded during a period of $100 \mathrm{~s}$ with a $50 \mathrm{~Hz}$ sampling rate using a high-resolution 24-bit 'Data Translation' DT9821 acquisition board. The internal pre-amplifier of the board with the gain of 64 ensured a $20 \mathrm{nV}$ 'noise-free' resolution in voltage at about the same RMS value of the noise. Besides the instrumental noise, the accuracy of measurements was affected also by thermally induced zero drift of the acquisition board. This effect was minimized by keeping the acquisition board in a thermo-box, which limited the total uncertainty of the electric potential measurement to $450 \mathrm{nV}$. For the sensitivity of the probe in the linear regime $0.2 \mu \mathrm{V} / \mathrm{mm} / \mathrm{s}$ (Fig. 3),

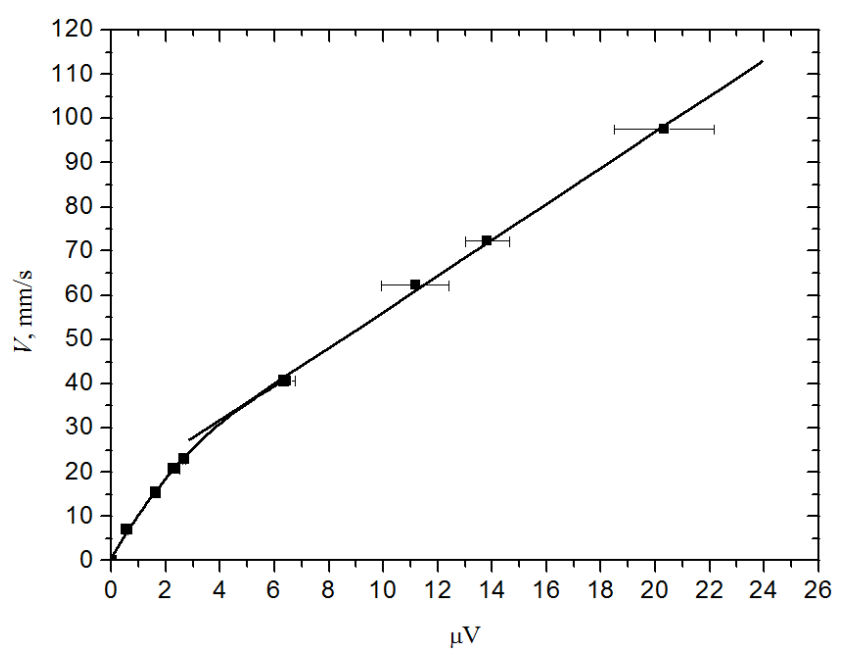

Fig. 3 Calibration curve for the potential probe with horizontal bars showing the standard deviation of the signal induced by the oscillations of the flow around the probe in the calibration channel.

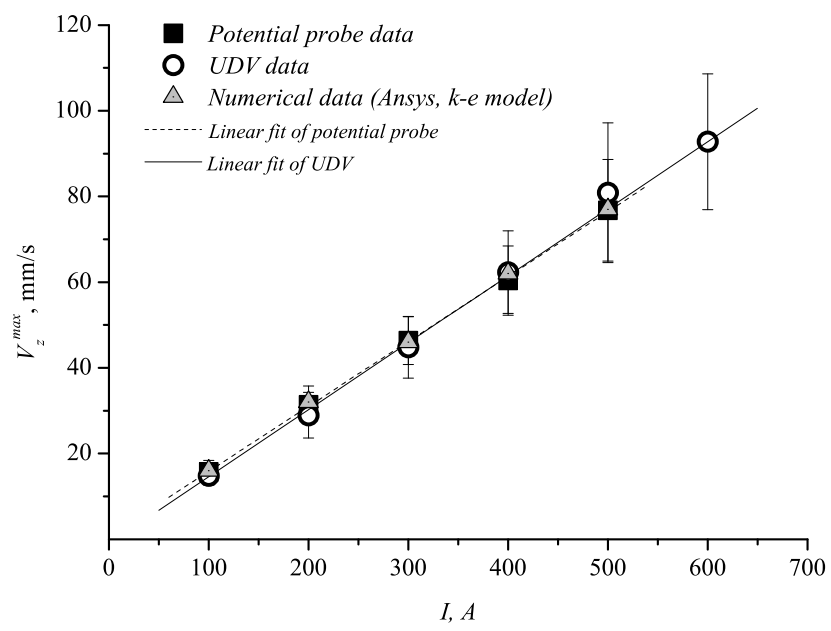

Fig. 5 Maximal axial velocity measured by the UDV technique (circles) and the potential probe (squares) along with numerical results (triangles) versus the effective current $I$ at the phase shift fixed to $\Delta \varphi=90^{\circ}$. The error bars show the standard deviation of turbulent velocity pulsations.

the uncertainty of the velocity measurements was about $2.3 \mathrm{~mm} / \mathrm{s}$.

\section{Experimental results}

\subsection{UDV measurements}

The basic effect of the phase shift between the currents in the top and bottom coils on the flow structure is illustrated by Fig. 4 which shows the isolines of the vertical velocity component for the magnetic field travelling upwards (a) and downwards (b). Note that the velocities are recorded as positive 


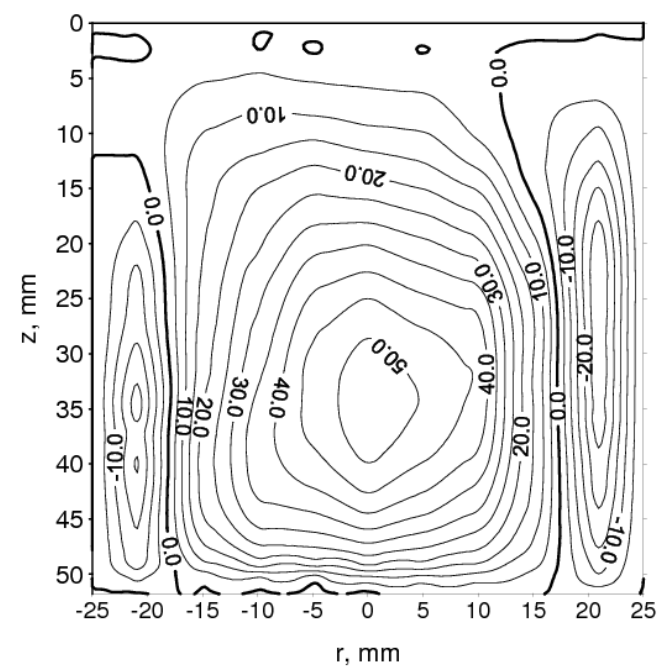

(a)

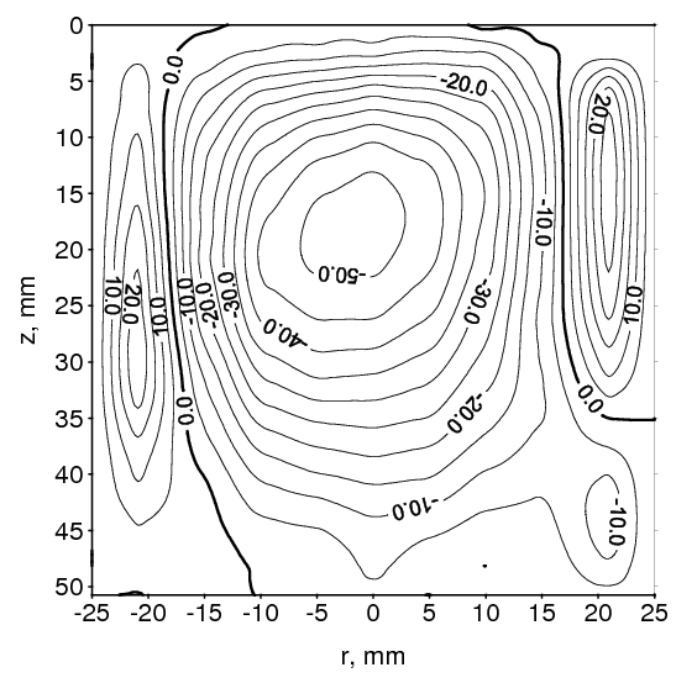

(b)

Fig. 4 Isolines of the axial velocity component for the magnetic field travelling downwards (a) and upwards (b) which correspond to the current in the upper coil lagging and leading, respectively, in phase by $90^{\circ}$ relative to the current in the bottom coil. In both coils the effective current is the same and equal to $I=350 \mathrm{~A}$.

when the flow is directed away, that is downward from the UDV probe which is placed at the top of the liquid metal. Also, note that the velocity could be measured by the UDV only up to $|r| \leq 21 \mathrm{~mm}$ and is then extrapolated to the region near the side wall where it is supposed to obey the no-slip condition. In both cases, the currents in the top and bottom coils alternate with the same frequency of $f=400 \mathrm{~Hz}$ and have the same effective values equal to $I=350 \mathrm{~A}$. The only difference is that in the first case, the current in the upper coil lags in phase by $90^{\circ}$ while in the second case, it leads by the same amount relative to the current in the bottom coil. As the lagging current follows the leading one, the magnetic field effectively travels from the former to the latter. And as it does so, it drags the liquid metal near the side wall with it. Respectively, the magnetic field travelling upward gives rise to regions of negative axial velocity at the side walls, while there is a larger region of positive velocity in the central part of the container where a downward axial jet forms. The highest axial velocity is attained in the jet at the symmetry axis $(r=0)$ somewhat downstream from the mid-height of the container. Switching the sign of the phase shift and thus the vertical direction in which the magnetic field travels swaps both the direction of the flow and its pattern about the midheight of the container. The slight axial asymmetry of the flow pattern observable in both cases may be due to the magnetic field perturbations by the current connections.
Two series of measurements were performed using the UDV. In the first series, the effective current $I$ in both coils was increased simultaneously from 100 to 600 A while the phase shift between them was kept fixed at $\Delta \varphi=90^{\circ}$. The maximal axial velocity, which for this case is plotted in Fig. 5, is seen to increase with the current almost linearly from 15 to $92 \mathrm{~mm} / \mathrm{s}$. Such a linearity, which results from the balance of electromagnetic and nonlinear inertial forces [14], is characteristic for turbulent ACdriven flows [16]. In laminar but strongly nonlinear flow regime, velocity is expected to vary as $\sim I_{0}^{4 / 3}[5$, 15].

Along with the velocity grow also its fluctuations which characterize the level of turbulence in the flow. The flow pattern remains basically similar to that shown in Fig. 4(left panel) with a slight axial asymmetry.

In the second series, the effective currents in both coils were kept fixed at $I=350 \mathrm{~A}$ while the phase shift $\Delta \varphi$ between them was increased from $0^{\circ}$ to $180^{\circ}$. Figure 6 shows the isolines of the axial velocity component measured at four different phase shifts $\Delta \varphi$ in addition to that of $\Delta \varphi=90^{\circ}$, which is plotted in Fig. 4 a. The first case with $\Delta \varphi=0^{\circ}$ shown in Fig. 6 a corresponds to a standard single-phase AC magnetic field. This field gives rise to a nearly compressing electromagnetic force with a maximum at the mid-height between the coils, where it drives the liquid metal radially inwards. As a result the typical two-vortex structure, which can be inferred from Fig. 6 a , arises. As seen in Fig. $6 \mathrm{~b}$, a phase shift of 

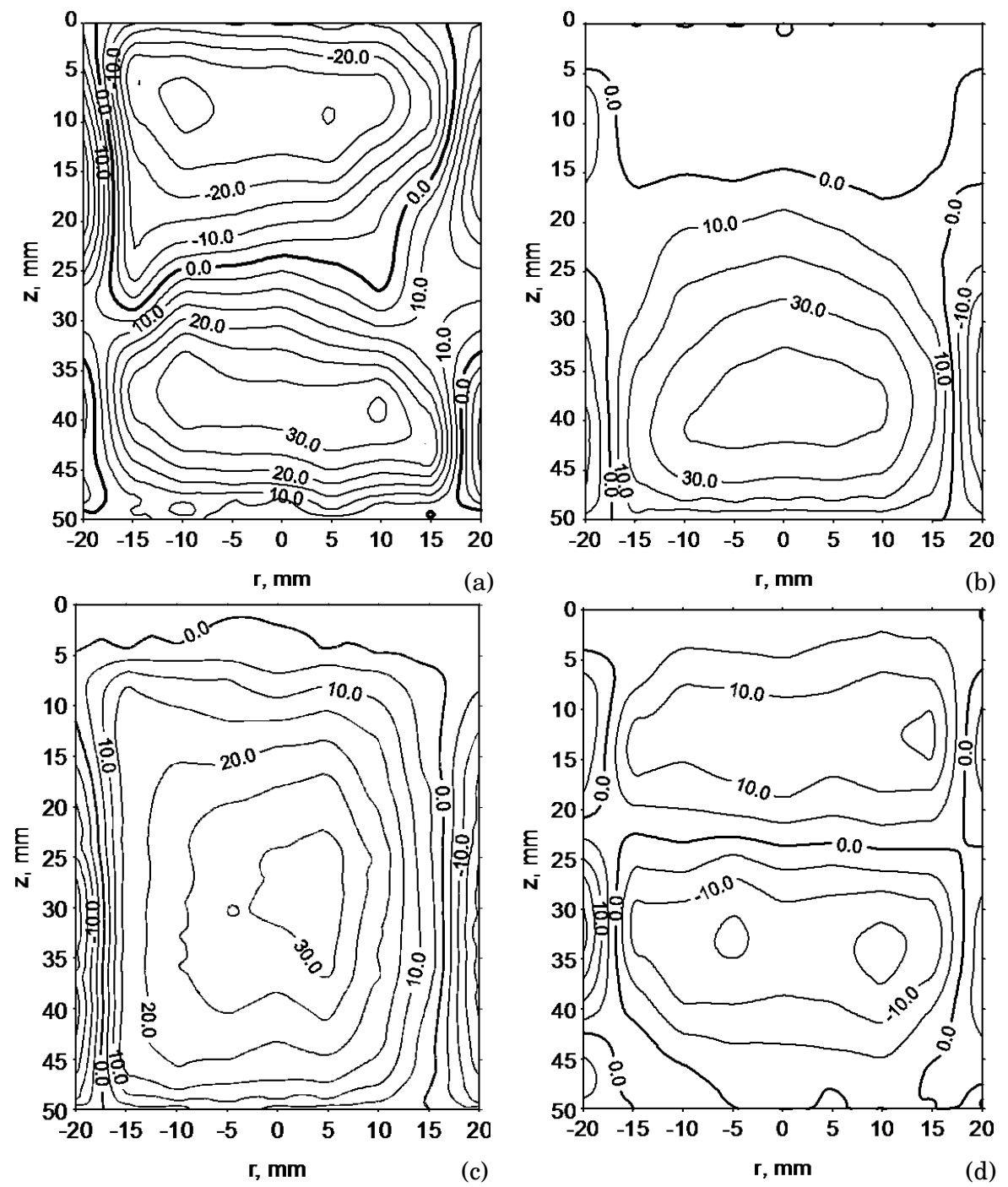

Fig. 6 Isolines of the axial velocity component for various phase shifts $\Delta \varphi$ between the currents in top and bottom coils (a) $0^{\circ}$, (b) $30^{\circ}$, (c) $120^{\circ}$, (d) $180^{\circ}$. The case of $\Delta \varphi=90^{\circ}$ can be seen in Fig. 4 (a)

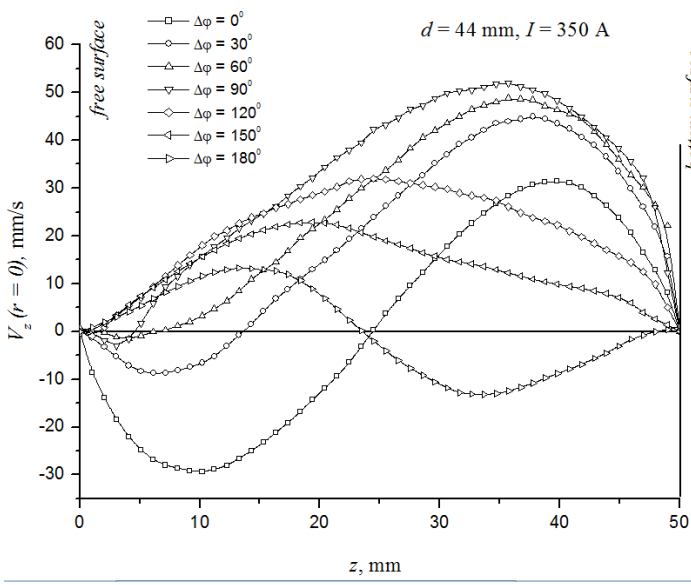

(a)

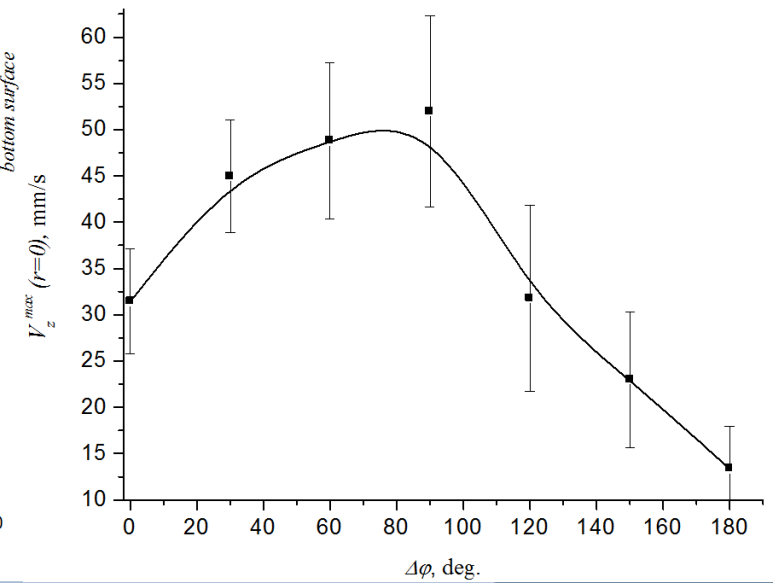

(b)

Fig. 7 Axial velocity profiles along the symmetry axis $(r=0)$ at different phase shifts between the currents in both coils (a). The highest axial velocity along the symmetry axis and the RMS of the associated turbulent velocity fluctuations versus the phase shift between the currents (b). 
$\Delta \varphi=30^{\circ}$ is sufficient to break this two-vortex balance in favour of the bottom one which is enhanced by the arising upward axial force while the top vortex is suppressed by it. The axial flow keeps increasing with the phase shift up to $\Delta \varphi=90^{\circ}$ where according to Fig. 7 it attains a maximum and then starts to decrease when the phase shift is increased further. The lowest axial velocity is attained at $\Delta \varphi=$ $180^{\circ}$ which again corresponds to a single-phase AC magnetic field. In contrast to the previous case, the currents in both coils now flowing in the opposite directions tend to cancel out each other's magnetic field. Thus, the electromagnetic pinching force cancels out in the mid-plane between the coils where the liquid is now pushed by the pressure gradient radially outwards. This gives rise to a two-vortex structure shown in Fig. 6d, which is similar to that for $\Delta \varphi=0^{\circ}$ seen in Fig. 6 6 axcept for the opposite flow direction and a twice lower velocity.

\subsection{Potential probe measurements}

Potential probe was used only in one case for $I=$ $350 \mathrm{~A}$ and $\Delta \varphi=90^{\circ}$, which was then compared with the UDV measurements and numerical results. The measured velocity field and the isolines of axial velocity component are shown in Fig. 8, The latter is seen to agree well with the corresponding UDV measurements shown in Fig. 4 a. Even the slight axial asymmetry is reproduced. This probe could reach the radial positions up to $r= \pm 23 \mathrm{~mm}$ so approaching the side wall closer than the UDV well into the region of the ascending flow. The full flow pattern shown in Fig. 8 includes an extrapolation of the velocity distribution up to the side walls with no-slip boundary condition applied. As seen in Fig. 5, there is also a good agreement between the maximal values of axial velocities measured by the potential and UDV probes.

\section{Comparison with numerical results}

The problem was also modelled numerically using the finite element code ANSYS in axially symmetric approximation. First, the ANSYS Emag solver was used to solve the electromagnetic part of the problem. At this stage, the effect of liquid metal flow on the AC magnetic field was neglected following the inductionless approximation which is commonly used in the liquid metal magnetohydrodynamics. The cross-section of metal was discretized using a rectangular uniform grid with $50 \times 100$ elements in ra-

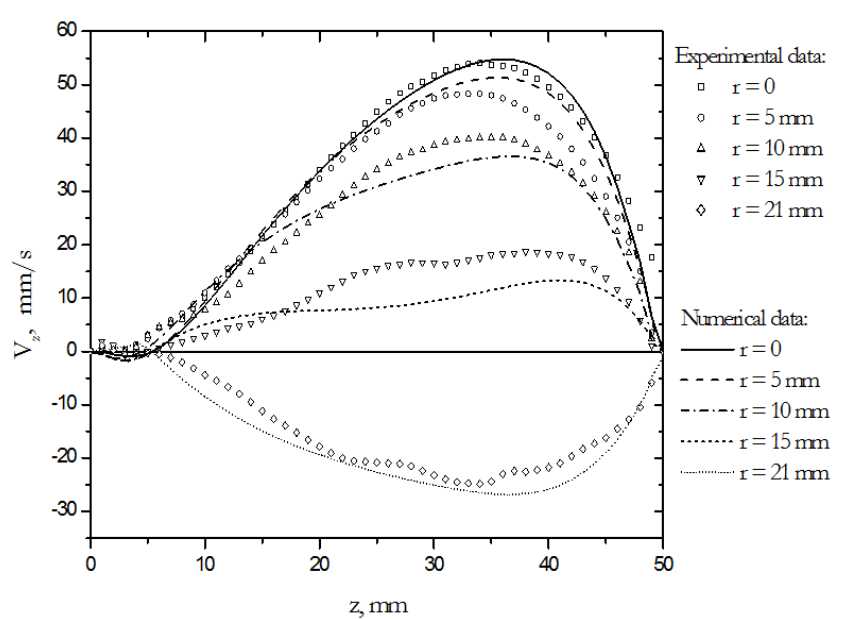

Fig. 9 Measured (UDV) and computed profiles of the mean axial velocity along the height of the container at various radii for $I=350 \mathrm{~A}$ and $\Delta \varphi=90^{\circ}$. Maximal RMS value of turbulent velocity pulsations is shown in Fig. 7bb).

dial and axial directions, respectively. The distribution of the magnetic field was computed in both the liquid metal and the surrounding areas for various coil currents and their phase shifts. From those data, the distribution of the electromagnetic force in the liquid metal was computed and then passed on as a body force to the FLOTRAN hydrodynamic solver of ANSYS. Using this solver with a standard $k-\varepsilon$ model of turbulence and no-slip boundary conditions at the container walls, the flow of liquid metal driven by AC magnetic field was computed using the same grid for the melt region.

First, as seen in Fig. 5, the numerical results demonstrate a good agreement for the maximal values of the axial velocity measured by the UDV technique at various currents and a phase shift fixed to $\Delta \varphi=90^{\circ}$. There is also a reasonable agreement between the measured and computed profiles of the axial velocity along the height of the container at various radii, which are plotted for $I=350 \mathrm{~A}$ and $\Delta \varphi=90^{\circ}$ in Fig. 9. The slight axial asymmetry in the measured velocity distribution, which may be due to the current connectors, was eliminated in the comparison by taking the mean value between positive and negative radii.

\section{Conclusion}

The velocity distribution in the flow of GaInSn eutectic alloy driven by a two-phase inductor in a cylindrical container was measured by ultrasonic Doppler velocimetry and local potential probes. The flow was also computed numerically, and a reasonable agree- 


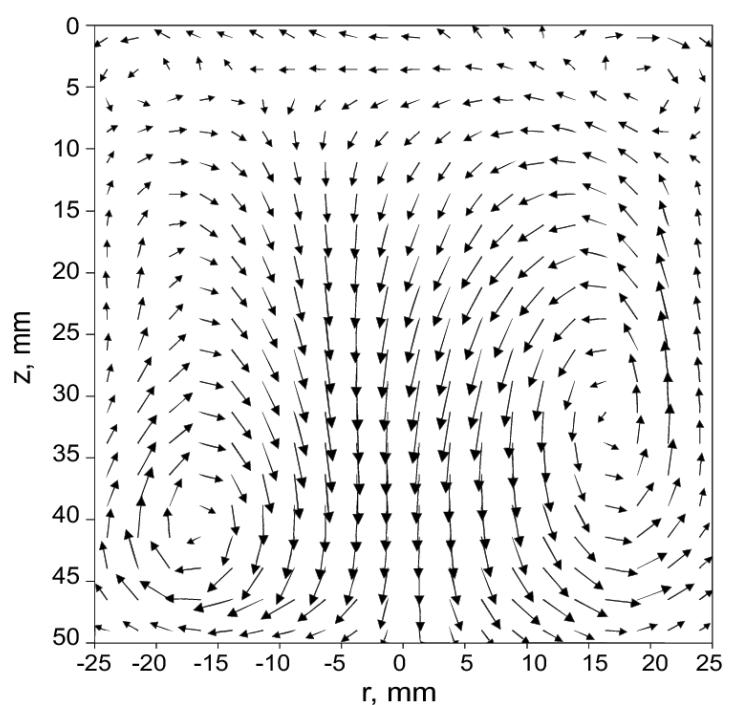

(a)

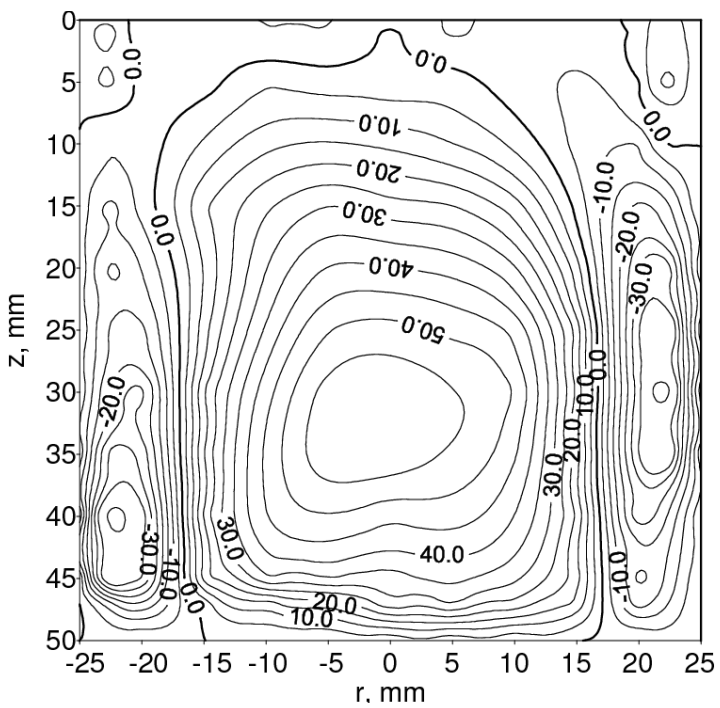

(b)

Fig. 8 The velocity field in the vertical cross-section (a) and the isolines of the axial velocity component (b) measured by the potential probe at $I=350 \mathrm{~A}$ and $\Delta \varphi=90^{\circ}$.

ment with the experimental results was found. It was demonstrated that the flow structure can be modified from the typical two toroidal vortices into a single vortex when the currents in the two coils are made to alternate with a certain phase shift. The vortex is strongest for a given current amplitude when the phase shift between the currents in both coils is about $90^{\circ}$. The obtained results may be useful for the design of combined two-phase electromagnetic stirrers and induction heaters for metal or semiconductor melts in applications where a well-defined stirring is required.

\section{References}

1. Cramer A, Zhang C, Eckert S (2005) Local flow structures in liquid metals measured by ultrasonic Doppler velocimetry. Flow Meas Instrum 15: 145-153

2. Cramer A, Varshney K, Gundrum Th, Gerbeth G (2006) Experimental study on the sensitivity and accuracy of electric potential local flow measurements. Flow Meas Instrum 17: 1-11

3. Ernst R, Perrier D, Brun P, Lacombe J (2005) Multiphase electromagnetic stirring of low conducting liquids. COMPEL 24:334-343

4. Ernst R, Hamburger J, Garnier C, Mangelinck-Noël N, Ramoni P (2005) Grain size reduction by electromagnetic stirring inside gold alloys. Eur Phys J Appl Phys 30: 215222

5. Fautrelle Y R (1982) Analytical and numerical aspects of the electromagnetic stirring induced by alternating magnetic fields. J Fluid Mech 102: 405-430

6. Hermann R, Priede J, Behr G, Gerbeth G, Schultz L (2001) Influence of growth parameters and melt convection on the solid-liquid interface during $R F$-floating zone crystal growth of intermetallic compounds. J Cryst Growth 223:577-587
7. Hermann R, Behr G, Gerbeth G, Priede J, Uhlemann H-J, Fischer F, Schultz L (2005) Magnetic field controlled FZ single crystal growth of intermetallic compounds. J Cryst Growth 275:1533-1538

8. Moffatt H K (1991) Electromagnetic stirring. Phys Fluids A 3: $1336-1343$

9. Priede J, Gerbeth G (2013) Analysis of two-phase RF inductor for the floating-zone crystal growth, COMPEL (submitted)

10. Priede J, Gerbeth G, Hermann R, Behr G, Schultz L and Uhlemann H-J (2007) German Patent DE10328859B4

11. Ricou R, Vives C (1982) Local velocity and mass transfer measurements in molten metals using an incorporated probe. Int J Heat Mass Transfer 25: 1579-1588

12. Rudolph $P$ (2008) Travelling magnetic fields applied to bulk crystal growth from the melt: The step from basic research to industrial scale. J Cryst Growth 310: 12981306

13. Rudolph $P$ (2010) Defect formation during crystal growth from the melt. In: Dhanaraj G, Byrappa K, Prasad V, Dudley M. (eds) Springer Handbook of Crystal Growth, 1st edn, Springer, Berlin, pp 159-201

14. Sneyd A D, Moffatt H K (1982) Fluid dynamical aspects of the levitation melting. J Fluid Mech 117: 45-70

15. Sneyd A D (1993) Theory of electromagnetic stirring by AC fields. IMA J Math Appl Bus Indust 5: 87-113

16. Taberlet E, Fautrelle Y (1985) Turbulent stirring in an experimental induction furnace. J Fluid Mech 159: 409431

17. Takeda Y (1987) Measurement of velocity profile of mercury flow by ultrasound Doppler shift method. Nucl Techn 79: 120-124

18. Takeda Y, Kikura H (2002) Flow mapping of the mercury flow, Exp. Fluids 32: 161-169 